\title{
A note on latent heat release from disequilibrium phase transformations and deep seismogenesis
}

\author{
Craig R. Bina \\ Department of Geological Sciences, Northwestern University, Evanston, Illinois, U.S.A.
}

(Received May 8, 1998; Revised October 19, 1998; Accepted October 23, 1998)

\begin{abstract}
Latent heat release by equilibrium mineralogical transformations in an adiabatically subducting slab reversibly perturbs temperatures and pressures so as to conserve entropy. However, latent heats of metastable transformations in such a slab yield irreversible isobaric temperature changes which increase entropy despite adiabatic constraints. As a result, latent heat release by metastable exothermic transformations can yield local superheating above the background adiabat, with the degree of potential superheating increasing with extent of metastable overstep. In real slabs, however, regions of metastably persisting low-pressure phases should undergo conductive warming from surrounding transformed material. Such warming should proceed more rapidly than warming of the bulk slab from the surrounding mantle, and the resulting decrease in metastable transition pressures will slightly decrease the degree of local superheating. Nonetheless, such local temperature increases may trigger seismic release of accumulated strain energy via a number of proposed mechanisms of shear instability. Adiabatic instability, in the form of shear localization in material with temperature-dependent rheology, is one mechanism which may be triggered by such latent heat release in metastable regions yet produce rupture that extends beyond the boundaries of such regions.
\end{abstract}

\section{Introduction}

A variety of phenomena, such as thermal deflection of phase boundaries or viscosity stratification, may contribute to significant stresses within subducting lithospheric slabs (Goto et al., 1987; Vassiliou and Hager, 1988; Bina, 1996, 1997; Yoshioka et al., 1997). Release of the associated strain energy in the form of deep seismicity, however, requires a mechanism that will allow slip to occur despite the large confining pressures prevailing at depth (Green and Houston, 1995). A number of mechanisms for such shear instabilities have been proposed, including dehydration, strain concentration in material with temperature-dependent rheology, formation of weak zones due to fine-grained material arising from phase transition or dynamic recrystallization, and runaway phase transformation arising from latent heat release (Bridgman, 1945; Kirby et al., 1991; Rubie and Ross, 1994; Green and Houston, 1995; Green and Zhou, 1996; Karato, 1997). Each of these mechanisms requires either localized heating or a mineralogical phase transition (under equilibrium or metastable conditions) or both.

Here I review the temperature effects associated with latent heat release from phase transformations under both equilibrium and metastable conditions in idealized adiabatically subducting slabs, noting that metastable exothermic transformations under such conditions can yield anomalously large local temperature increases, and I comment on the extent to which useful work may thereby be obtained. I then introduce the effects of conductive heating of small regions of material that may have persisted metastably beyond their stability

Copy right (C) The Society of Geomagnetism and Earth, Planetary and Space Sciences (SGEPSS); The Seismological Society of Japan; The Volcanological Society of Japan; The Geodetic Society of Japan; The Japanese Society for Planetary Sciences. field, noting that such heating slightly decreases the degree of local superheating associated with metastable exothermic transition. Finally, I consider the extent to which possible triggering of seismicity by such local superheating, due to latent heat release during transformation of metastable phases, may be consistent with observed patterns of deep seismicity.

\section{Subduction along an Adiabat}

\subsection{Equilibrium transformations}

The $P-T$ path of idealized slab material subducting along an adiabat (i.e., assuming no heat exchange with adjacent material) follows the standard adiabatic gradient:

$$
\left(\frac{\partial T}{\partial P}\right)_{S}=\frac{T \alpha V}{C_{P}}
$$

where $T$ is temperature, $V$ volume, $C_{P}$ isobaric heat capacity, and $\alpha$ volume coefficient of thermal expansion. When the material undergoes an equilibrium phase transformation, such as the exothermic $\alpha \rightarrow \beta$ transition in olivine near $400 \mathrm{~km}$ depth, the adiabat is deflected along the equilibrium boundary of the phase transition (Fig. 1) in accordance with the Clapeyron relation:

$$
\frac{d T}{d P}=\frac{\Delta V^{\alpha \rightarrow \beta}}{\Delta S^{\alpha \rightarrow \beta}}
$$

where $\Delta V$ and $\Delta S$ are the changes in volume and entropy, respectively, across the phase transition. This refraction of the adiabat, which is based upon the assumption (discussed below) that rates of reaction and attendant latent heat release significantly exceed rates of conductive heat transfer, results in a net temperature and pressure increment across the phase 


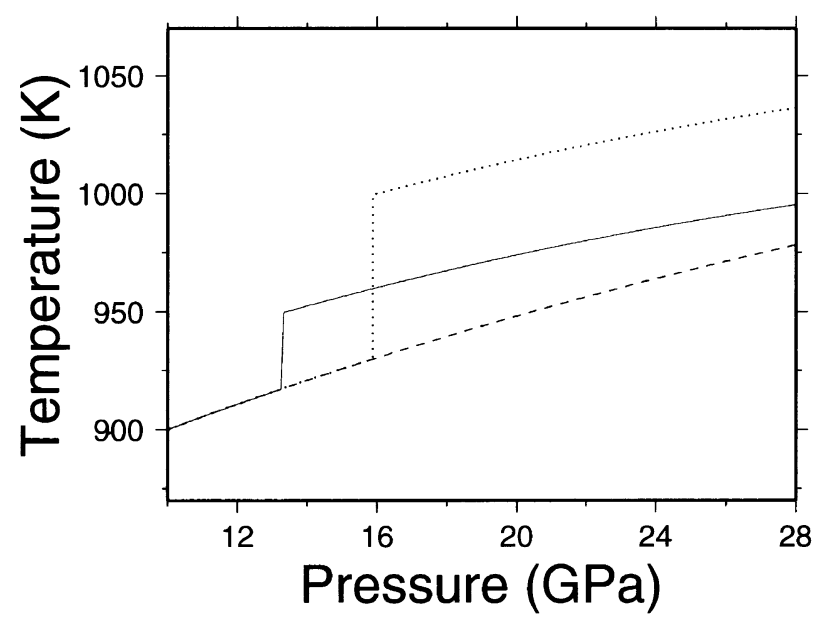

Fig. 1. Temperature as a function of pressure for a portion of a lithospheric slab subducting along an adiabat that is refracted by an equilibrium exothermic phase transition (solid). Regions in which low pressure phases persist metastably continue to follow their own adiabat (dashed). Eventual metastable transformation (here at $930 \mathrm{~K}$ ) yields isobaric superheating (dotted) above the background equilibrium adiabat. Diagram calculated for the $\alpha \rightarrow \beta$ transition in forsterite.

transition:

$$
\begin{gathered}
\Delta T \approx \frac{-\Delta H^{\alpha \rightarrow \beta}}{C_{P}-\frac{T \alpha V \Delta S^{\alpha \rightarrow \beta}}{\Delta V^{\alpha \rightarrow \beta}}}=\frac{-T \Delta S^{\alpha \rightarrow \beta}}{C_{P}-\frac{T \alpha V \Delta S^{\alpha \rightarrow \beta}}{\Delta V^{\alpha \rightarrow \beta}}}, \\
\Delta P \approx \frac{\Delta S^{\alpha \rightarrow \beta}}{\Delta V^{\alpha \rightarrow \beta}} \Delta T \approx \frac{-T\left(\Delta S^{\alpha \rightarrow \beta}\right)^{2}}{C_{P} \Delta V^{\alpha \rightarrow \beta}-T \alpha V \Delta S^{\alpha \rightarrow \beta}}
\end{gathered}
$$

where $C_{P}, \alpha$, and $V$ are for the high-pressure phase. These increments are often approximated as:

$$
\Delta T \sim \frac{-T \Delta S^{\alpha \rightarrow \beta}}{C_{P}}, \quad \Delta P \sim \frac{-T\left(\Delta S^{\alpha \rightarrow \beta}\right)^{2}}{C_{P} \Delta V^{\alpha \rightarrow \beta}}
$$

and called the "Verhoogen effect" (Jeanloz and Thompson, 1983).

The $\Delta T$ value given by the expression (5) for the Verhoogen effect simply reflects the conversion of the latent heat of equilibrium phase change $(\Delta H=T \Delta S)$ into temperature via the isobaric heat capacity. However, since the reaction possesses a finite Clapeyron slope, such heating at equilibrium cannot be isobaric, and the more complete expression (3) for $\Delta T$ above reflects the additional contribution of adiabatic compression. These increments in $T$ and $P$ serve to maintain adiabaticity (i.e., constant entropy $S$ ) within the slab across the transition.

\subsection{Metastable transformations}

In the non-equilibrium case, $\alpha$ persists metastably into the stability field of $\beta$, because temperatures within the cold slab material are insufficient to overcome the activation energy barrier to transformation (Sung and Burns, 1976a,b). Metastably persistent $\alpha$ will continue to follow its own adiabat, given by Eq. (1), which remains colder than the refracted adiabat of the transformed material. In this case, when the transformation eventually does occur, it takes place in the absence of such equilibrium constraints as the Clapeyron equation (2) above, and it results in simple isobaric heating:

$$
\Delta T \approx \frac{-\Delta H}{C_{P}}=\frac{-(T \Delta S+\Delta G)}{C_{P}}
$$

where $G$ is the Gibbs free energy, in which the latent heat of phase change induces a temperature change through the isobaric heat capacity.

Thus, $\Delta H$ is the relevant isobaric potential for heat in both equilibrium and metastable processes (Klotz, 1964). However, the expression for $\Delta H$ in the metastable case (6) differs from that in the equilibrium case (3) by $\Delta G$, which is identically zero at equilibrium but negative for metastable transformation. The effect upon $\Delta H$ of metastable postponement of the transition can be described in terms of the oversteps in pressure and temperature:

$$
d \Delta H(P, T)=[\Delta V-T \Delta(\alpha V)] d P+\Delta C_{P} d T
$$

where the pressure dependence has occasionally been approximated by omission of the $\alpha V$ term (Rubie and Ross, 1994; Kirby et al., 1996). Both expressions (6) and (7) show that an exothermic subsolidus phase transition under metastable conditions yields a greater latent heat release than under equilibrium conditions (Fig. 1), an effect which also has been observed in numerical simulations (Daessler and Yuen, 1993; Daessler et al., 1996). Furthermore, a greater degree (i.e., depth) of metastable persistence, corresponding to greater $\Delta G$ of eventual transition, will generate a greater $\Delta T$ and so greater local heating above the slab background.

Thus, exothermic latent heat release in an adiabatically subducting slab yields local superheating by raising the temperature of any metastable material above the level of the ambient adiabat upon eventual transformation. While equilibrium transformation is a reversible process in which $S$ remains constant under adiabatic conditions, metastable transformation is an irreversible process in which a net increase in $S$ occurs despite adiabatic conditions preventing heat exchange with the surroundings (Klotz, 1964).

Since the Gibbs free energy change of reaction, $\Delta G$, is zero for equilibrium transformation but negative for metastable transformation, it has been suggested that additional useful work may be available from the latter (Kirby et al., 1996). Indeed, $G$ is a potential for the net work $W_{\text {net }}$ reversibly performed by the system at constant pressure and temperature, exclusive of the necessary $P d V$ work of expansion or contraction:

$$
W_{\text {net,irrev }}<W_{\text {net, rev }}=-(\Delta G)_{P, T}
$$

where the work performed irreversibly is always less than the reversible value, and the heat $Q$ liberated by the system will be correspondingly reduced:

$$
Q=-\Delta H-W_{\text {net }}
$$

thereby reducing the degree of local superheating. However, to the extent that the reaction rates of irreversible transformation exceed the characteristic rates of any processes for recovering useful work, such transformations are likely to approach the limit of explosively irreversible processes in which $W_{\text {net }}$ is zero. 
Since most studies involving latent heats of mantle phase transformations have assumed, explicitly or implicitly, that reaction rates of transformation significantly exceed both rates of heat conduction and rates of work recovery, some discussion of this assumption may be in order. Exothermic olivine polymorphic transformations that occur under conditions of significant metastable overstep exhibit rapid growth (Brearley and Rubie, 1994; Kubo et al., 1998). For the $\alpha \rightarrow \beta$ transformation, two completing primary mechanisms have been revealed experimentally. The first mechanism involves the incoherent nucleation of $\beta$ on grain boundaries of $\alpha$ and exhibits interface-controlled growth (Rubie and Ross, 1994; Kerschhofer et al., 1996). Kinetically, greater overstep of the equilibrium boundary results in a smaller required critical nucleus (Burnley, 1995), and the larger driving force $(\Delta G)$ for transformation enhances both nucleation and growth rates (Rubie and Ross, 1994). Furthermore, the small activation volume (Kubo et al., 1998) falls with increasing pressure (Rubie and Ross, 1994), becoming negative at large overstep pressures (Burnley, 1995), thus enhancing the growth rate by decreasing the effective activation energy (Rubie and Ross, 1994). The second mechanism involves coherent intracrystalline nucleation of $\gamma$ lamellae on shear-induced stacking faults followed by nucleation of $\beta$ on the $\gamma$ (Kerschhofer et al., 1996; Chai et al., 1998). For this mechanism, which may be more important in cold subducting slabs (Burnley, 1995; Kerschhofer et al., 1996), a large pressure overstep enhances the shear-induced transformation (Burnley, 1995; Kerschhofer et al., 1996), both because greater overstep of the equilibrium boundary provides a greater driving force for transformation (Burnley, 1995) and because smaller strain energies are required to form $\gamma$ lamellae at high pressures due to the greater compressibility of $\alpha$ (Burnley, 1995).

All of the above effects conspire to elevate reaction rates for olivine transformations under conditions of large metastable overstep. For transformation under near-equilibrium conditions, on the other hand, the small driving force $(\Delta G)$ of transition will depress reaction rates. Furthermore, the $\alpha \rightarrow \beta$ transformation exhibits diffusion-controlled growth under such conditions (Rubie and Ross, 1994), and the consequent necessity for diffusion of cations through bulk crystals rather than simply across grain boundaries will further depress the kinetics of near-equilibrium transformation. Thus, the conditions under which the assumption of rapid rates of reaction (relative to rates of thermal conduction) is likely to be most accurate are precisely those prevailing in the case of metastably persistent phases.

\subsection{Multiple transformations}

The examples thus far have featured a single phase transformation. In complex multicomponent systems such as the mantle, however, a series of multiple phase transformations may occur with increasing depth. In mantle olivine, for example, the exothermic $\alpha \rightarrow \beta$ transition is followed by the exothermic $\beta \rightarrow \gamma$ and endothermic $\gamma \rightarrow p v+m w$ transitions. Tabulated thermodynamic parameters for these phases (Fei et al., 1991) allow determination of the relevant pressure-temperature paths for the associated phase transformations, where for simplicity these have been calculated for pure forsterite (Fig. 2) rather than for a forsterite-90 solid

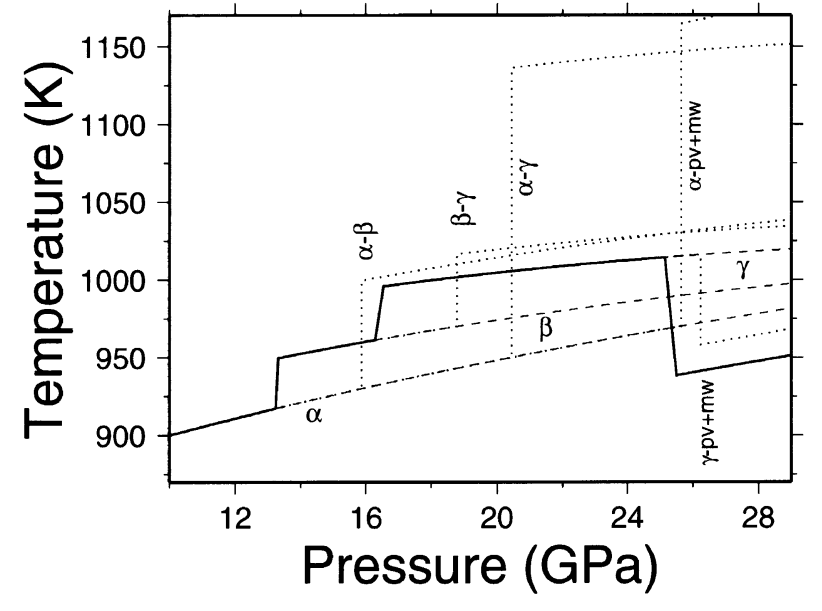

Fig. 2. Temperature as a function of pressure for a portion of a lithospheric slab subducting along an adiabat that is refracted by multiple equilibrium phase transitions (solid). Regions in which low pressure phases $(\alpha, \beta, \gamma)$ persist metastably continue to follow their own adiabats (dashed). Eventual metastable transformations $(\alpha \rightarrow \beta, \beta \rightarrow \gamma$, $\alpha \rightarrow \gamma, \alpha \rightarrow p v+m w, \gamma \rightarrow p v+m w$ ) yield isobaric superheating (dotted) above the background equilibrium adiabat. Diagram calculated for transitions in forsterite.

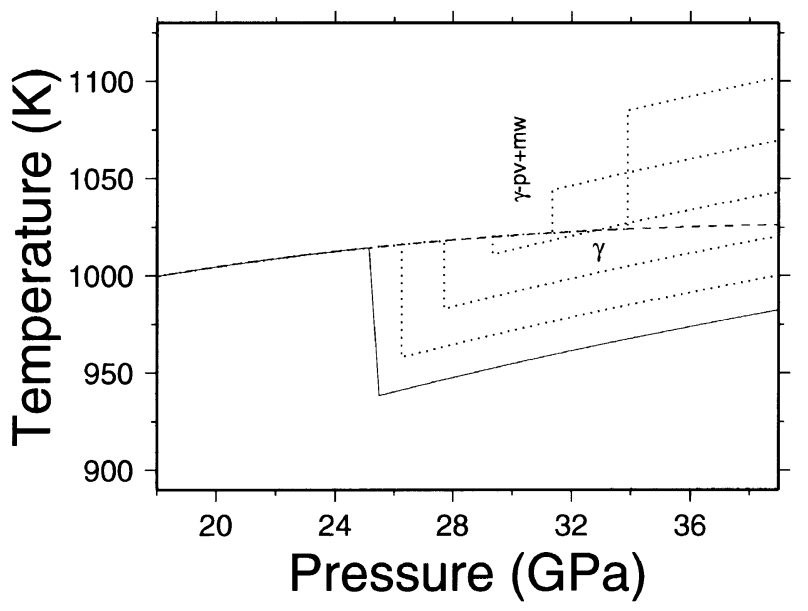

Fig. 3. Temperature as a function of pressure for a portion of a lithospheric slab subducting along an adiabat that is refracted by an endothermic equilibrium phase transition (solid). Regions in which the low pressure phase $(\gamma)$ persists metastably continue to follow their own adiabat (dashed). Eventual metastable transformation $(\gamma \rightarrow p v+m w)$ initially yields isobaric cooling (dotted). However, the transformation becomes exothermic at higher pressures, according to Eq. (7), thus eventually yielding isobaric superheating (dotted). Diagram calculated for transitions in forsterite.

solution. The metastable $\alpha \rightarrow \beta$ and $\beta \rightarrow \gamma$ transitions are exothermic and so yield local superheating above the ambient adiabat. The metastable $\alpha \rightarrow \gamma$ transition, being essentially a combination of the previous two, is significantly more exothermic and so yields a greater degree of local superheating. The metastable $\alpha \rightarrow p v+m w$ transition is also strongly exothermic, yielding local superheating.

On the other hand, the metastable $\gamma \rightarrow p v+m w$ transition is endothermic and so yields local cooling. However, 
the metastable $\gamma \rightarrow p v+m w$ transition, while initially endothermic, becomes exothermic at higher pressures (Fig. 3) due to the dominance of the $\Delta V$ term in Eq. (7). This behavior, too, is derived from a standard thermochemical database (Fei et al., 1991), but better constraints may be provided by more recent experimental data on the thermochemistry of the post- $\gamma$ phase transitions (Akaogi et al., 1998).

Moreover, transitions among phases other than the olivine polymorphs, such as those between pyroxenes and garnetmajorite solid solutions, may also exhibit disequilibrium behavior under subduction zone conditions. Enstatite, for example, has been shown to persist metastably at low temperatures (Hogrefe et al., 1994). The latent heats of such additional transitions will also perturb the local temperature structure. Furthermore, the presence of one phase may alter the metastable behavior of another. The stability of highpressure clinopyroxenes in the mantle (Woodland, 1998), for example, may serve to enhance the kinetics of the olivine transformations (Sharp and Rubie, 1995).

\section{Subduction with Heat Conduction}

\subsection{Exothermic transformations}

While an idealized subducting slab may be considered to transform under equilibrium conditions and so to follow an adiabat refracted along the phase boundary as discussed above, in reality its edges will warm by conduction from the surrounding mantle. Moreover, any regions of $\alpha$ which persist metastably into the $\beta$ field (or subsequent fields) will also warm by conduction from the surrounding transformed slab material, rather than continuing along their own adiabat as in the idealized adiabatic case above. In both cases, the temperature of the colder material will approach that of the surrounding warmer material with a thermal lag $\lambda$ (Fig. 4) which depends upon time $t$ and distance from the edge $y$ as follows:

$$
\lambda(y, t) \approx \frac{2 \Delta T}{\pi} \sum_{n=1}^{\infty} e^{-\left(\frac{n \pi}{L}\right)^{2} \kappa t}\left[\frac{1-(-1)^{n}}{n}\right] \sin \frac{n \pi y}{L}
$$

where $\Delta T$ is the initial temperature difference between the two materials and $L$ is the characteristic spatial extent of the warming region (Pinsky, 1991). The characteristic time $\tau$ required for such material to be substantially heated above its adiabat thus depends upon spatial extent as $L^{2}$. Hence, a $10-\mathrm{km}$-wide region of metastable material, for example, will rise in temperature by conduction from the surrounding hotter slab material approximately 100 times faster than the $100-\mathrm{km}$-thick bulk slab will heat by conduction from the surrounding mantle.

As a result, when a region of metastably persisting material does undergo an exothermic phase transformation, its initial temperature will already have risen somewhat above its adiabat by conduction from surrounding transformed material. However, if transformation is triggered by attaining a critical temperature, the material will attain that temperature at a lower pressure than would material which adhered to the adiabat. The net result, via Eq. (7), is a slight decrease in the degree of local superheating attending metastable transformation (Fig. 4). Again, greater depths of metastable persistence will generate greater degrees of local superheating.

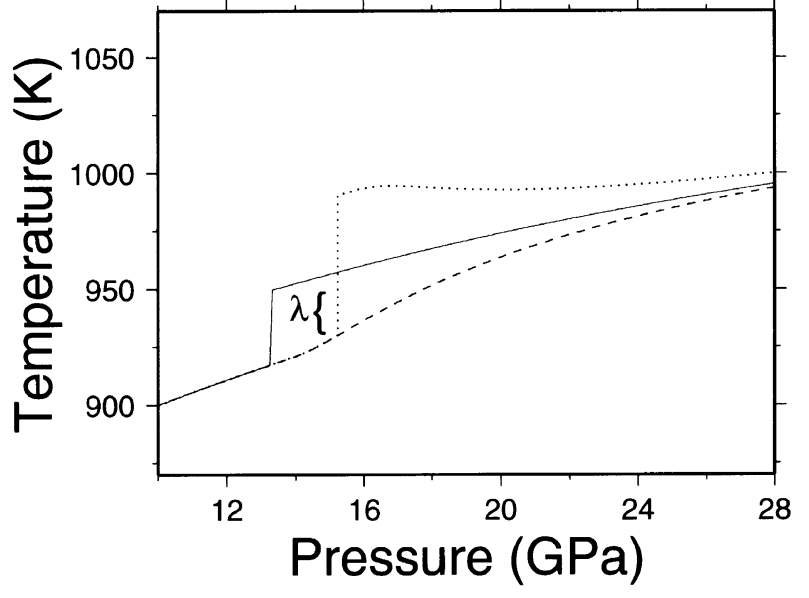

Fig. 4. Temperature as a function of pressure for a portion of a lithospheric slab subducting along an adiabat that is refracted by an equilibrium exothermic phase transition (solid). Regions in which low pressure phases persist metastably continue to follow their own geotherm (dashed), but this departs from an adiabat due to conductive heating from the surrounding warmer transformed material with a thermal lag $\lambda$ according to Eq. (10). Eventual metastable transformation (here at $930 \mathrm{~K}$ ) yields isobaric superheating (dotted) above the background equilibrium adiabat. Diagram calculated for the $\alpha \rightarrow \beta$ transition in forsterite, for $30-\mathrm{km}$-diameter metastable regions with $6 \mathrm{~cm} / \mathrm{yr}$ vertical descent rate.

\subsection{Endothermic transformations}

In the case of an endothermic phase transition (e.g., $\gamma \rightarrow$ $p v+m w$ near $670 \mathrm{~km}$ depth), any metastably persisting material would find itself surrounded by colder slab material, and subsequent heat conduction would lower its temperature (Green and Houston, 1995; Green and Zhou, 1996) with the same sort of lag $\lambda$ as described (10) above. Subsequent endothermic transformation of the metastable material would also yield cooling, which would be unlikely to effectively trigger a seismogenic mechanism involving thermal runaway. However, conductive heating of slab material from the surrounding mantle would eventually overcome such cooling, so that other seismogenic mechanisms (such as dehydration) would remain possible at greater depths (Green and Houston, 1995; Okal and Bina, 1998). Moreover, a sufficient degree of metastable overstep of an endothermic phase boundary may cause the metastable transformation to become exothermic (Fig. 3).

\section{Discussion and Conclusions}

In summary, the temperature of metastable material that subducts adiabatically should, upon eventual exothermic transformation, rise via latent heat effects above the adiabatic temperature of the transformed bulk material surrounding it. However, regions of metastable material will not subduct strictly adiabatically, since they will warm by conduction from the hotter transformed material surrounding them (Devaux et al., 1997), and the resulting decrease in transformation pressure will slightly reduce the effective magnitudes of the latent heats. Nonetheless, eventual transformation of metastable material can yield local superheating above the temperature of the surrounding material, with greater depths of metastable persistence generating greater 
degrees of local superheating. Such superheating could trigger release of strain energy through a variety of proposed mechanisms of seismogenic shear instability, such as dehydration, runaway phase transformation (possibly with attendant crystallization of fine-grained weak material), or temperature-dependent rheological weakening. Regardless of the particular mechanisms of shear instability, thermal control of the failure process is consistent with the observation that the statistical properties of deep earthquakes and their aftershock sequences exhibit a greater temperature dependence than do those of shallow earthquakes, with colder slabs exhibiting a greater frequency of small earthquakes as well as more substantial aftershock sequences for deep earthquakes (Wiens and Gilbert, 1996).

It is instructive to contrast two proposed mechanisms in the context of latent heat release. In the runaway phase transformation model, latent heat release enhances reaction kinetics in neighboring metastable material via conductive heating, giving rise to locally rapid reaction rates (Kirby et al., 1991; Rubie and Ross, 1994; Green and Houston, 1995; Green and Zhou, 1996; Kirby et al., 1996). Such a failure mechanism, however, cannot operate outside the region of metastable material, contrary to observations constraining the actual rupture extent of deep seismicity (Wiens et al., 1994; Myers et al., 1995; Silver et al., 1995).

In the temperature-dependent rheological weakening model, on the other hand, shear localization occurs through ductile deformation. Consequent shear heating, arising from concentrated conversion of elastic strain energy (RegenauerLieb and Yuen, 1998), leads to weakening in material with temperature-dependent rheology. This further focuses additional shear deformation, and such a feedback cycle leads to "adiabatic instability" as a potential mechanism for deep seismicity (Ogawa, 1987; Hobbs and Ord, 1988; Karato, 1997). Indeed, in addition to this subsolidus temperatureinduced rheological weaking, such shear localization may induce partial melting (Ogawa, 1987; Karato, 1997) leading to even greater local reductions in viscosity. While there is no direct evidence of such melting in the transition zone, shear localization appears in some cases to have induced high degrees of partial melting within the lithosphere (Austrheim and Boundy, 1994; Obata and Karato, 1995; Austrheim et al., 1996; Jin et al., 1998), and frictional melting has also been suggested recently as an adjunct to deep seismicity (Kanamori et al., 1998). Local superheating arising from latent heat release due to disequilibrium transformation of metastable phases could initiate such adiabatic instability by locally depressing viscosities within the superheated zone, and consequent shear localization could induce failure beyond the extent of the metastable material in which it was initially triggered.

Furthermore, it is interesting to compare the anomalous heating behavior expected for a series of phase transitions in a subducting slab and the observed distribution of deep seismicity (Green and Houston, 1995). Increasingly metastable persistence of $\alpha$ below an uplifted (exothermic) $\alpha \rightarrow \beta$ transition in the slab is consistent with increasing seismicity with depth below $400 \mathrm{~km}$, and onset of a depressed (endothermic) $\gamma \rightarrow p v+m w$ transition is consistent with cessation of seismicity below $700 \mathrm{~km}$. Of the various metastable olivine transformations considered herein (Fig. 2), those involving the metastable persistence of $\alpha$, rather than of $\beta$ or $\gamma$, may be the most important in subducting slabs, because the lowest temperatures in the slab are found initially within the $\alpha$ stability field. Of these, the metastable $\alpha \rightarrow \gamma$ transition yields the greatest latent heat release in the upper mantle.

If $\alpha$ can persist metastably into the lower mantle, then the metastable $\alpha \rightarrow p v+m w$ transition will release even greater latent heat. Also, any metastable persistence of $\gamma$ leads to a $\gamma \rightarrow p v+m w$ reaction that, while initially endothermic, grows progressively more exothermic at greater depths in the lower mantle (Fig. 3). Thus, if lower pressure phases can persist metastably below the depth of the equilibrium $\gamma \rightarrow p v+m w$ transition, a mechanism for generating local superheating can also operate below this depth, and the apparent absence of seismicity in the lower mantle (Okal and Bina, 1998) may simply be due to a dearth of high strain energies below the transition zone (Bina, 1997).

Finally, because conductive heating of the slab occurs initially at the edges rather than in the interior, the edge regions initially possess a greater potential for local thermal anomalies due to metastable transformations, possibly consistent with occasional observation of double Wadati-Benioff zones at intermediate depths (Wiens et al., 1993). However, heating of the slab edges leaves little scope for metastable persistence in these regions at greater depths, and the large degree of overstep associated with any metastable areas in the cold slab interior confers upon this region the greatest potential for initiation of seismicity at large depths.

Acknowledgments. I am grateful to the personnel of Tokyo University's Earthquake Research Institute for their kind hospitality and to the U.S. National Science Foundation (INT-9603234 and EAR9706152) for financial support. I thank Harry Green, Fred Marton, and Dave Rubie for helpful comments on the manuscript.

\section{References}

Akaogi, M., H. Kojitani, K. Matsuzaka, and T. Suzuki, Postspinel transformations in the system $\mathrm{Mg}_{2} \mathrm{SiO}_{4}-\mathrm{Fe}_{2} \mathrm{SiO}_{4}$ : Element partitioning, calorimetry, and thermodynamic calculation, in Properties of Earth and Planetary Materials at High Pressure and Temperature, edited by $\mathrm{M} . \mathrm{H}$ Manghnani and T. Yagi, pp. 373-384, American Geophysical Union, Washington, D.C., 1998.

Austrheim, H. and T. M. Boundy, Eclogite facies pseudotachylytes from the Bergen Arcs, western Norway: Records of rapid faulting and seismicity during eclogitization of the deep crust, Science, 265, 82-83, 1994.

Austrheim, H., M. Erambert, and T. M. Boundy, Garnets recording deep crustal earthquakes, Earth Planet. Sci. Lett., 139, 223-238, 1996.

Bina, C. R., Phase transition buoyancy contributions to stresses in subducting lithosphere, Geophys. Res. Lett., 23, 3563-3566, 1996.

Bina, C. R., Patterns of deep seismicity reflect buoyancy stresses due to phase transitions, Geophys. Res. Lett., 24, 3301-3304, 1997.

Brearley, A. J. and D. C. Rubie, Transformation mechanisms of San Carlos olivine to $(\mathrm{Mg}, \mathrm{Fe})_{2} \mathrm{SiO}_{4} \beta$-phase under subduction zone conditions, Phys. Earth Planet. Inter., 86, 45-67, 1994.

Bridgman, P. W., Polymorphic transitions and geological phenomena, Am. J. Sci., 243a, 90-97, 1945

Burnley, P. C., The fate of olivine in subducting slabs: A reconnaissance study, Amer. Mineral., 80, 1293-1301, 1995.

Chai, M., J. M. Brown, and Y. Wang, Yield strength, slip systems and deformation induced phase transition of San Carlos olivine up to transition zone pressure at room temperature, in Properties of Earth and Planetary Materials at High Pressure and Temperature, edited by M. H. Manghnani and T. Yagi, pp. 483-494, American Geophysical Union, Washington, D.C., 1998.

Daessler, R. and D. A. Yuen, The effects of phase transition kinetics on subducting slabs, Geophys. Res. Lett., 20, 2603-2606, 1993. 
Daessler, R., D. A. Yuen, S. Karato, and M. R. Riedel, Two-dimensional thermo-kinetic model for the olivine-spinel phase transition in subducting slabs, Phys. Earth Planet. Inter., 94, 217-239, 1996.

Devaux, J. P., G. Schubert, and C. Anderson, Formation of a metastable olivine wedge in a descending slab, J. Geophys. Res., 102, 24,627-24,637, 1997.

Fei, Y., H.-K. Mao, and B. O. Mysen, Experimental determination of element partitioning and calculation of phase relations in the $\mathrm{MgO}-\mathrm{FeO}-\mathrm{SiO}_{2}$ system at high pressure and high temperature, J. Geophys. Res., 96, $2157-$ 2169, 1991.

Goto, K., Z. Suzuki, and H. Hamaguchi, Stress distribution due to olivinespinel phase transition in descending plate and deep focus earthquakes, J. Geophys. Res., 92, 13,811-13,820, 1987.

Green, H. W., II and H. Houston, The mechanics of deep earthquakes, Annu. Rev. Planet. Sci., 23, 169-213, 1995.

Green, H. W., II and Y. Zhou, Transformation-induced faulting requires an exothermic reaction and explains the cessation of earthquakes at the base of the mantle transition zone, Tectonophys., 256, 39-56, 1996.

Hobbs, B. E. and A. Ord, Plastic instabilities: Implications for the origin of intermediate and deep focus earthquakes, J. Geophys. Res., 93, 10,52110,540, 1988

Hogrefe, A., D. C. Rubie, T. G. Sharp, and F. Seifert, Metastability of enstatite in deep subducting lithosphere, Nature, 372, 351-353, 1994.

Jeanloz, R. and A. B. Thompson, Phase transitions and mantle discontinuities, Rev. Geophys. Space Phys., 21, 51-74, 1983.

Jin, J., S.-I. Karato, and M. Obata, Mechanisms of shear localization in the continental lithosphere: Inference from the deformation microstructures of peridotites from the Ivrea zone, northwestern Italy, J. Struct. Geol., 20 195-209, 1998.

Kanamori, H., D. L. Anderson, and F. H. Heaton, Frictional melting during the rupture of the 1994 Bolivian earthquake, Science, 279, 839-842, 1998

Karato, S.-I., Phase transformations and rheological properties of mantle minerals, in Earth's Deep Interior: The Doornbos Memorial Volume, edited by D. J. Crossley, pp. 223-272, Gordon and Breach, Amsterdam, 1997.

Kerschhofer, L., T. G. Sharp, and D. C. Rubie, Intracrystalline transformation of olivine to wadsleyite and ringwoodite under subduction zone conditions, Science, 274, 79-81, 1996.

Kirby, S. H., W. B. Durham, and L. Stern, Mantle phase changes and deepearthquake faulting in subducting slabs, Science, 252, 216-225, 1991.

Kirby, S. H., S. Stein, E. A. Okal, and D. C. Rubie, Metastable mantle phase transformations and deep earthquakes in subducting oceanic lithosphere, Rev. Geophys., 34, 261-306, 1996.

Klotz, I. M., Chemical Thermodynamics, 468 pp., W. A. Benjamin Inc., New York, 1964

Kubo, T., E. Ohtani, T. Kato, H. Morishima, D. Yamazaki, A. Suzuki, K. Mibe, T. Kikegawa, and O. Shimomura, An in situ X ray diffraction study of the $\alpha-\beta$ transformation kinetics of $\mathrm{Mg}_{2} \mathrm{SiO}_{4}$, Geophys. Res. Lett., 25 , 695-698, 1998.

Myers, S. C., T. C. Wallace, S. L. Beck, P. G. Silver, G. Zandt, J. VanDecar, and E. Minaya, Implications of spatial and temporal development of the aftershock sequence for the $M_{w}$ 8.3 June 9, 1994 deep Bolivia earthquake, Geophys. Res. Lett., 22, 2269-2272, 1995.

Obata, M. and S.-I. Karato, Ultramafic pseudotachylite from the Balmuccia peridotite, Ivrea-Verbano zone, northern Italy, Tectonophys., 242, 313328, 1995.

Ogawa, M., Shear instability in a viscoelastic material as the cause of deep focus earthquakes, J. Geophys. Res., 92, 13,801-13,810, 1987.

Okal, E. A. and C. R. Bina, On the cessation of seismicity at the base of the transition zone, J. Seism., 2, 65-86, 1998.

Pinsky, M. A., Partial Differential Equations and Boundary-Value Problems with Applications, 461 pp., McGraw-Hill, New York, 1991.

Regenauer-Lieb, K. and D. A. Yuen, Rapid conversion of elastic energy into plastic shear heating during incipient necking of the lithosphere, Geophys. Res. Lett., 25, 2737-2740, 1998.

Rubie, D. C. and C. R. Ross, II, Kinetics of the olivine-spinel transformation in subducting lithosphere: Experimental constraints and implications for deep slab processes, Phys. Earth Planet. Inter, 86, 223-241, 1994.

Sharp, T. G. and D. C. Rubie, Catalysis of the olivine to spinel transformation by high-clinoenstatite, Science, 269, 1095-1098, 1995.

Silver, P. G., S. L. Beck, T. C. Wallace, C. Meade, S. C. Myers, D. E. James, and R. Kuehnel, Rupture characteristics of the deep Bolivian earthquake of 9 June 1994 and the mechanism of deep-focus earthquakes, Science, 268, 69-73, 1995 .

Sung, C.-M. and R. G. Burns, Kinetics of the olivine-spinel transition: Implications to deep-focus earthquake genesis, Earth Planet. Sci. Lett., 32, 165-170, 1976a.

Sung, C.-M. and R. G. Burns, Kinetics of the high-pressure phase transformations: Implications to the evolution of the olivine-spinel phase transition in the downgoing lithosphere and its consequences on the dynamics of the mantle, Tectonophys., 31, 1-32, 1976b.

Vassiliou, M. S. and B. H. Hager, Subduction zone earthquakes and stress in slabs, Pure Appl. Geophys., 128, 547-624, 1988.

Wiens, D. A. and H. J. Gilbert, Effect of slab temperature on deep-earthquake aftershock productivity and magnitude-frequency relations, Nature, 384, 153-156, 1996.

Wiens, D. A., J. J. McGuire, and P. J. Shore, Evidence for transformational faulting from a deep double seismic zone in Tonga, Nature, 364, 790-793, 1993

Wiens, D. A., J. J. McGuire, P. J. Shore, M. G. Bevis, K. Draunidalo, G. Prasad, and S. P. Helu, A deep earthquake aftershock sequence and implications for the rupture mechanism of deep earthquakes, Nature, 372, 540-543, 1994.

Woodland, A. B., The orthorhombic to high-P monoclinic phase transition in Mg-Fe pyroxenes: Can it produce a seismic discontinuity?, Geophys. Res. Lett., 25, 1241-1244, 1998

Yoshioka, S., R. Daessler, and D. A. Yuen, Stress fields associated with metastable phase transitions in descending slabs and deep-focus earthquakes, Phys. Earth Planet. Inter., 104, 345-361, 1997.

C. R. Bina (e-mail: craig@earth.nwu.edu) 